\title{
Analysis of EEG-EMG Coherence in Low Frequency Bands
}

\author{
Krishnamoorthy ARUNGANESH ${ }^{\mathrm{a}, 1}$, Natarajan SIVAKUMARAN ${ }^{\mathrm{a}}$, \\ Shanmugasundaram KUMARAVEL ${ }^{\mathrm{b}}$ and Pa KARTHICK ${ }^{\mathrm{a}}$ \\ ${ }^{a}$ Department of Instrumentation and Control Engineering, National Institute of \\ Technology, Tiruchirappalli, Tamilnadu, India \\ ${ }^{b}$ Department of Orthopedics, Thanjavur Medical College, Tamilnadu, India
}

\begin{abstract}
In this, study, an attempt is made to analyze the corticomuscular coupling of the brain and muscular system in the low-frequency components during ramp descent (RD) and stair descent (SD) locomotion. For this purpose, magnitude squared coherence (MSC) is computed from the simultaneous EEG and EMG signals recorded during the ramp and stair descent tasks. The MSC is extracted from the low- frequency bands such as delta $(0.1-3 \mathrm{~Hz})$ and theta bands $(4-7 \mathrm{~Hz})$. The study utilizes a publicly available database consisting of simultaneous recorded EEG, lower limb EMG and full body motion information from ten healthy subjects. The results show that there exists corticomuscular coupling between motor cortex $\left(\mathrm{C}_{1}, \mathrm{C}_{2}\right.$ and $\mathrm{C}_{\mathrm{z}}$ contacts $)$ and tibialis anterior muscle activities during $\mathrm{RD}$ and $\mathrm{SD}$. In addition, the MSC differs for both the tasks and frequency bands. In delta band frequencies, the MSC is found to be higher in $\mathrm{C}_{2}$ regions. In the case of theta, the MSC is higher in $\mathrm{C}_{1}$ during $\mathrm{RD}$ and in $\mathrm{C}_{\mathrm{z}}$ during $\mathrm{SD}$. Therefore, the MSC associated with the low frequency components could be used to detect walking intentions.
\end{abstract}

Keywords. Corticomuscular activity, magnitude squared coherence.

\section{Introduction}

Human movement and its control involve a complex relationship between the brain and the muscles [1]. The functional connection and interactions between these systems are analyzed using the coherence function and it is performed using the magnitude squared coherence. Analysis of the coherence is found to be useful in the treatment of dyskinesia, and the development of active rehabilitation devices. In this work, the magnitude square coherence of simultaneously recorded electroencephalography (EEG) and electromyography (EMG) are investigated in the low frequency bands [2].

\section{Methods}

The study used the publicly available full body mobile brain-body imaging data [1]. The database consists of simultaneously recorded EEG and EMG activities during five steady locomotion, namely, level ground walking, stair descent, stair ascent, ramp descent and

\footnotetext{
${ }^{1}$ Corresponding Author, Department of Instrumentation and Control Engineering, National Institute of Technology, Tiruchirappalli, Tamilnadu, India. ; E-mail: arunganeshkg@gmail.com.
} 
ascent. The activities were recorded at a sampling rate of $1000 \mathrm{~Hz}$ from ten healthy participants. For this analysis, we only considered the tasks, namely, ramp descent (RD) and stair descent (SD). The magnitude squared coherence (MSC) is used to determine the corticomuscular coupling between the EEG signals and EMG signals of tibialis anterior (TA) muscle. The mathematical expression for the MSC is given by,

$$
M S C_{x y}(\mathrm{f})=\frac{\left[\mathrm{S}_{\mathrm{xy}}(\mathrm{f})\right]^{2}}{\mathrm{~S}_{\mathrm{xx}}(\mathrm{f}) * \mathrm{~S}_{\mathrm{yy}}(\mathrm{f})}
$$

Where $S_{x y}$ is the cross-correlation of EEG and EMG signal. The parameters, $S_{x x}$ and $S_{\mathrm{yy}}$ are the autocorrelations of EEG and EMG respectively. The coherence is expressed as a real number between 0 and 1, with 1 indicating a perfect linear association [2]. The MSC is evaluated for the delta $(0.1-3 \mathrm{~Hz})$ and theta frequency bands $(4-7 \mathrm{~Hz})$.

\section{Results and Discussion}
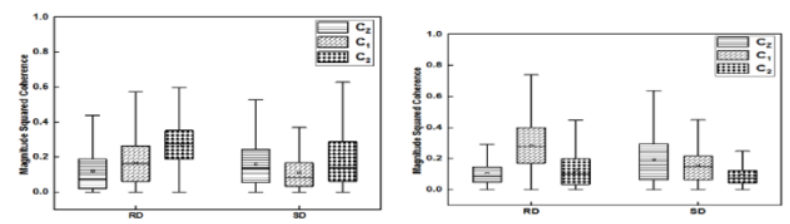

Figure 1. MSC of simultaneous EEG and EMG signals associated with (a) delta (0.1-3 Hz, and (b) theta frequency band $(4-7 \mathrm{~Hz})$ during stair and ramp descent tasks.

The variation of MSC in the low-frequency components namely, delta and theta frequency bands are illustrated in figure 1 . The electrode contacts $C_{1}, C_{2}$ and $C_{z}$ correspond to the motor cortex region of the brain. The results suggest that there is a significant corticomuscular coupling between the motor cortex and TA muscle. The coherence between the $\mathrm{C}_{2}$ region of the brain and EMG activities is found to be higher in the delta band frequencies during both $\mathrm{RD}$ and $\mathrm{SD}$ tasks. Besides the $\mathrm{C}_{2}$, the coupling is higher in $\mathrm{C}_{1}$ than in the $\mathrm{C}_{z}$ region during $\mathrm{RD}$. In the case of the $\mathrm{SD}$ events, the coherence in $\mathrm{C}_{Z}$ is higher than in the $\mathrm{C}_{1}$ region. In the Theta band frequency, the median value of coherence is higher in the $\mathrm{C}_{1}$ region during $\mathrm{RD}$ and in the $\mathrm{C}_{z}$ region during SD. It is to be noticed that the degree of corticomuscular coupling is considerably lower in the $\mathrm{C}_{2}$ region under the $\mathrm{SD}$ task. This result indicates that there exists a task-specific modulation in the corticomuscular coupling. Therefore, the MSC measure along with machine learning algorithms could be used for the detection of motor intent in neuromuscular and neurological patients. This will enable researchers and scientists to develop active rehabilitation and assistive devices.

\section{References}

[1] Brantley JA, Luu, TP, Nakagome S, Zhu F, Contreras-Vidal JL.Full body mobile brain-body imaging data during unconstrained locomotion on stairs, ramps, and level ground. Sci Data.2018;5:180133.

[2] Xugang X, Cunbin M, Changmin Y, Seyed M, Xian H,. Enhanced EEG-EMG coherence analysis based on hand movements. Biomedical signal processing and control. 2020;56:101727. 